\title{
Análise de conformidades em Unidades de Alimentação e Nutrição no Brasil
}

\author{
Compliance analysis in Food and Nutrition Units in Brazil \\ Análisis de cumplimiento en Unidades de Alimentación y Nutrición en Brasil
}

\author{
Stefano Sullivan Soares Viana \\ ORCID: https://orcid.org/0000-0003-2074-1343 \\ Centro Universitário Fametro, Brasil \\ E-mail: stefano.sullivan@yahoo.com \\ Lucas Fernandes Esquivel \\ ORCID: https://orcid.org/0000-0002-3148-1914 \\ Centro Universitário Fametro, Brasil \\ E-mail: esquivellucas35@gmail.com \\ Lucas Matheus Souza Campos \\ ORCID: https://orcid.org/0000-0002-4160-0884 \\ Centro Universitário Fametro, Brasil \\ E-mail: 1c857784@gmail.com \\ Francisca Marta Nascimento de Oliveira Freitas \\ ORCID: https://orcid.org/0000-0002-0044-0925 \\ Centro Universitário Fametro, Brasil \\ E-mail: Francisca.freitas@fametro.edu.br \\ Rebeca Sakamoto Figueiredo \\ ORCID: https://orcid.org/0000-0002-9819-8099 \\ Centro Universitário Fametro, Brasil \\ E-mail: rebeca.figueiredo@fametro.edu.br
}

\begin{abstract}
Resumo
Introdução: Com a finalidade de assegurar a qualidade da alimentação servida em uma Unidade de Alimentação e Nutrição (UAN), vários cuidados precisam ser adotados em razão do seu complexo funcionamento. Fazendo-se necessária a avaliação e monitoramento das condições de produção e manipulação dessas UAN's. A grande demanda do serviço de alimentação, o torna um segmento de grande relevância nacional. Nesse contexto é de fundamental importância que as unidades de alimentação sejam submetidas a avaliações da produção e manipulação de alimentos, a fim de garantir a qualidade higiênico-sanitária evitando as Doenças de Origem Alimentar (DOA). Objetivo: O objetivo do estudo foi realizar o levantamento dos índices de conformidades em diferentes serviços de alimentação no Brasil. Metodologia: Trata-se de uma estudo revisão integrativa de literatura (RIL), entre os anos de 2010 a 2020 em que foram selecionados artigos científicos que analisaram e classificou as conformidades de diversos serviços de alimentação no território brasileiro. Conclusão: Sendo assim o presente estudo observou-se que em média as Unidades de Alimentação e Nutrição de diferentes regiões do Brasil possuem condições higiênico-sanitária parcialmente adequadas. As não-conformidades ocorrem em todos os blocos analisados sendo maior a incidência naqueles que necessitam de registro documental. Conclui-se que se faz necessário que os órgãos de controle sanitário voltados para alimentação tenham melhor controle e rigor quanto à fiscalização dos registros, considerando que a alimentação ofertada nesses ambientes deve ser de qualidade sanitária e nutricional.
\end{abstract}

Palavras-chave: Conformidades; Serviços de alimentação; Controle higiênico-sanitário.

\begin{abstract}
Introduction: In order to ensure the quality of food served in a Food and Nutrition Unit (UAN), several precautions need to be taken due to its complex functioning. It is necessary to evaluate and monitor the conditions of production and handling of these UAN's. The great demand of the food service makes it a segment of great national relevance. In this context, it is of fundamental importance that the food units are submitted to evaluations of the production and handling of food, in order to guarantee the hygienic-sanitary quality, avoiding Foodborne Diseases (DOA). Objective: The aim of the study was to survey compliance rates in different food services in Brazil. Methodology: This is an integrative literature review (RIL) study, between the years 2010 to 2020, in which scientific articles that analyzed and classified the compliance of various food services in the Brazilian territory were selected. Conclusion: Therefore, in this study it was observed that, on average, the Food and Nutrition Units in different regions of Brazil have partially adequate hygienic-sanitary conditions. Non-conformities occur in all analyzed blocks, with a higher incidence in those that require documental registration. It is concluded that it is necessary that the sanitary control agencies focused on food have better control and strictness regarding the inspection of records, considering that the food offered in these environments must be of sanitary and nutritional quality.
\end{abstract}

Keywords: Compliance; Food services; Hygienic-sanitary, control. 


\begin{abstract}
Resumen
Introducción: Con una fuente de alimento, la calidad de los alimentos que se sirven en una Unidad de Alimentación y Nutrición (UAN), es necesario adoptar varios debido al funcionamiento del complejo. Es necesario evaluar y monitorear las condiciones de producción y manejo de estas UAN. La gran demanda del servicio de alimentación lo convierte en un segmento de gran oferta nacional. En este contexto, es de fundamental importancia que las unidades de alimentos sean sometidas a evaluaciones de la producción y manipulación de alimentos, con el fin de garantizar la calidad higiénico-sanitaria, evitando las Enfermedades Transmitidas por los Alimentos (DOA). Objetivo: El objetivo del estudio es sondear las tasas de cumplimiento en diferentes servicios alimentarios en Brasil. Metodología: Se trata de un estudio de revisión integradora de la literatura (EIR), entre los años 2010 a 2020, en el que se seleccionaron artículos científicos que analizaron y clasificaron como conformidad de diversos servicios alimentarios en el territorio brasileño. Conclusión: Por lo tanto, el presente estudio observó que, en promedio, las Unidades de Alimentación y Nutrición en diferentes regiones de Brasil tienen condiciones higiénico-sanitarias acreditadas. Dado que se producen no conformidades en todos los bloques de tratamiento, mayores que las que pertenecen al registro documental. Se concluye que es necesario que los organismos de control sanitario enfocados en alimentos tengan un mejor control y rigor en la inspección de registros, considerando que los alimentos en estos ambientes deben ser de calidad sanitaria y nutricional.
\end{abstract}

Palabras clave: Cumplimientos; Servicios de comida; Control higiénico-sanitario.

\title{
1. Introdução
}

A importância e a dimensão dessa área na economia nacional podem ser medidas a partir dos números gerados pelo segmento de alimentação (Aguiar, 2013). Segundo a Associação Brasileira de Refeições Coletivas (Aberc) (2019), que reúne as empresas prestadoras de serviços de alimentação, até o fim do ano de 2019, estimava que o fornecimento de mais de 21 milhões de refeições/dia, para instituições públicas ou privadas, gerando 250 mil empregos e um faturamento aproximado de 50 bilhões de reais.

Com o aumento no número de refeições oferecidas fora do lar, torna-se grande a preocupação com a qualidade dos alimentos oferecidos aos usuários. Milhões de pessoas são diretamente afetadas por Doenças de Origem Alimentar (DOA) em todo o mundo. Dados epidemiológicos mostram que as UAN figuram entre os principais locais de ocorrência de surtos de DTA, e que os fatores causadores estão diretamente ligados com o processo produtivo e como: práticas inadequadas executadas pelos manipuladores de alimentos (Rodrigues, 2010).

A busca pela qualidade em todos os setores da atividade humana é algo inerente ao ser humano. Especialmente para os alimentos, qualidade significa competência, profissionalismo e, sobretudo, competitividade e produtividade (Cintra, 2016; Vidal, 2011). Uma das estratégias para se atingir um alto padrão de qualidade é a implementação das Boas Práticas de Manipulação dos Alimentos (Sacool et al., 2012; Gobis, 2012).

No Brasil a responsabilidade maior pela implementação das ações que garantam a saúde e segurança dos consumidores de alimentos é inerente às autoridades reguladoras. Na área de alimentos os órgãos responsáveis pela legislação de alimentos são Ministério da Saúde, por meio da Agência Nacional de Vigilância Sanitária (Anvisa) e o Ministério da Agricultura, Pecuária e Abastecimento (MAPA) (Azeredo, 2017).

A legislação sanitária brasileira, através da Agência Nacional de Vigilância Sanitária (ANVISA), exige que os estabelecimentos produtores e/ou manipuladores de alimentos implantem procedimentos a fim de garantir a qualidade higiênico-sanitária dos alimentos como as Boas Práticas de Fabricação, controle APPCC (Análise de Perigos e Pontos Críticos de Controle), POP (Procedimento Operacional Padronizado) dentre outros (Brasil, 2004; Brasil, 2002). Nesse contexto a produção passou a ser conduzida com foco no controle da qualidade dos produtos através de ações direcionadas para por processos desde a entrada da matéria-prima na linha de produção até a saída do produto final (Jucene, 2019; Almeida, 2012).

Diante da importância do tema para a saúde da coletividade, e considerando o risco à saúde que as más condições higiênico-sanitárias em estabelecimentos produtores de alimentos representam, este estudo visou analisar as conformidades encontradas em diversas unidades de alimentação de nutrição no Brasil. 


\section{Metodologia}

Trata-se de um estudo tipo revisão integrativa de literatura (RIL) (Pereira et al., 2018). A qual seguiu as etapas estabelecidas por Mendes et al. (2008): estabelecimento de hipótese ou questão de pesquisa; busca na literatura; categorização dos estudos; interpretação dos resultados; síntese do conhecimento ou apresentação da revisão. Tendo como pergunta de partida a indagação: Qual o índice de conformidades higiênico-sanitárias dos serviços de alimentação no Brasil? Que tem a intenção de analisar artigos científicos quanto às conformidades em unidades produtoras de alimentos em diversas regiões do Brasil, entre os anos de 2010 a 2020.

Buscaram-se publicações indexadas na Biblioteca Virtual de Saúde (BVS) em três bases de dados: Scientific Eletronic Library (SCIELO), Medical Literature Analysis and Retriveal System Online (MEDLINE) e Literatura Latino Americana e do Caribe em Ciências da Saúde (LILACS). Utilizaram-se para buscas os seguintes indexadores "conformidades"; "serviços de alimentação"; "controle higiênico-sanitário". Adotaram-se como critérios de inclusão os artigos científicos completos, produzidos em território nacional, publicados entre os anos de 2010 a 2020 e em idioma como português. Dentre os critérios de exclusão: artigos científicos repetidos ou incompletos e resumos.

As informações extraídas dos autores foram: autor(es), ano, local do serviço de alimentação analisado e o percentual das categorias a saber: 1) Edificações, instalações, equipamentos, móveis e utensílios; 2) Higienização de equipamentos instalações, móveis e utensílios; 3) Controle integrado de vetores e pragas; 4) Abastecimento de água; 5) Manejo de Resíduos; 6) Manipuladores; 7) Matérias-primas, ingredientes e embalagens; 8) Preparo de alimentos; 9) Armazenamento e transporte de alimentos; 10) Documentação e registro; 11) Responsabilidade.

Inicialmente foram identificados 91 artigos, estes artigos passaram por uma etapa de seleção baseada nos critérios metodológicos adotados na pesquisa e, ao final desta etapa seletiva, pôde-se extrair 28 artigos a serem utilizados como base para a análise proposta neste estudo.

Adotou-se os critérios do Conselho Federal de Nutricionistas para estabelecer a classificação dos serviços de alimentação quanto ao tipo de estabelecimento. Para melhor avaliação, as categorias foram classificadas em três grupos, de acordo com os itens avaliados, estabelecendo: Grupo 1 (76\% - 100\%), Grupo 2 (51\% a 75\%) e Grupo 3 (< ou igual a 50\% de adequação) (Fonseca \& Santana, 2012).

\section{Resultados e Discussão}

\subsection{Atividades e serviços de alimentação}

O MAPA define Boas Práticas de Fabricação (BPF) como sendo os procedimentos necessários empregados para resultar numa produção de alimentos inócuos, saudáveis e sãos, estabelecidos os requisitos gerais e essenciais para a elaboração de alimentos elaborados/ industrializados para o consumo (MAPA, 1997). De forma geral, os itens que fazem parte do escopo das BPF são: limpeza e conservação das instalações; calibração de instrumentos; higiene pessoal; qualidade da água; qualidade das matérias-primas; recebimento e estocagem de matérias-primas controle integrado de pragas; e treinamento periódico de para funcionários (Fiori-Stangarlin, 2016; Brasil, 2004).

De acordo com a RDC $n^{\circ} 52$, define-se que os serviços de alimentação realizam algumas das seguintes atividades: manipulação, preparação, fracionamento, armazenamento, distribuição, transporte, exposição à venda e entrega de alimentos preparados para o consumo, caracterizados como cantinas, bufês, comissárias, confeitarias, cozinhas industriais, cozinhas institucionais, unidades de alimentação e nutrição dos serviços de saúde, delicatéssens, lanchonetes, padarias, pastelarias, restaurantes, rotisserias e congêneres (Brasil, 2014).

Nos serviços de alimentação as boas-práticas devem ser adotadas em todas as etapas de produção e ao se avaliar os riscos sanitários de uma UAN deve se classificar quanto a seu percentual de adequação em 3 categorias: GRUPO 1 - índice de 
adequação entre $76 \%$ a $100 \%$, GRUPO 2 - índice de adequação entre $75 \%$ a $51 \%$ e GRUPO 3 - índice de adequação < ou igual a 50\% (Sacool et al., 2012).

\subsection{Revisões literária: coleta e análise de dados de publicações}

Foram encontrados 91 artigos publicados na íntegra, após verificação observou-se que 28 artigos seguiam todos os critérios de pesquisa e os demais foram excluídos por não contemplarem aspectos metodológicos necessários para avaliação. O fluxograma segundo as diretrizes PRISMA (Preferred Reporting Items for Systematic Reviews and Meta-Analyses) detalhado na Figura 1.

Figura 1 - Fluxograma da seleção de artigos para revisão.

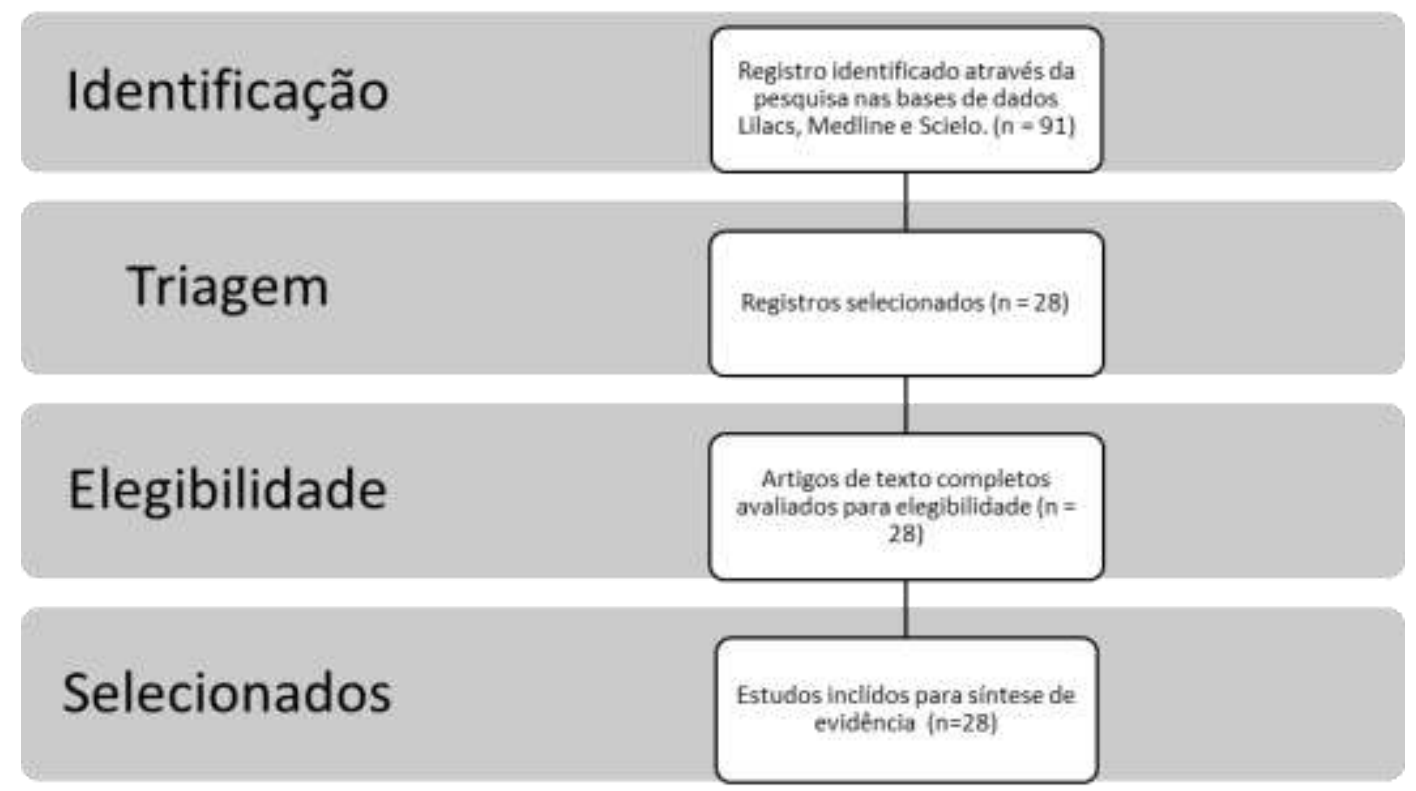

Fonte: Dados da pesquisa (2021).

Dentre os estudos coletados no período proposto foram avaliados 740 serviços de alimentação de diversos seguimentos: padarias, restaurantes, creche, UAN entre outros (Quadro 1). Estas unidades de alimentação foram encontradas variações percentuais acentuadas, variando entre $0 \%$ a $100 \%$ nos valores mínimos e máximos, respectivamente, conforme demonstra a Quadro 2. 
Quadro 1 - Estudos brasileiros sobre condições higiênicas de serviços de alimentação, conforme tamanho da amostra, descrição e Estado (2010 a 2020). Brasil, 2021.

\begin{tabular}{|c|c|c|c|c|}
\hline AUTOR & ANO & AMOSTRA (n) & DESCRIÇÃO & UF* \\
\hline Figueiredo et al. & 2013 & 10 & Comercias & MA \\
\hline Vasconcelos et al. & 2013 & 38 & Institucionais & $\mathrm{RJ}$ \\
\hline Miranda et al. & 2012 & 5 & Comerciais & $\mathrm{BA}$ \\
\hline Santos et al. & 2019 & 1 & Institucionais & $\mathrm{MG}$ \\
\hline Soares et al. & 2016 & 9 & Institucionais & RJ \\
\hline Costa et al. & 2016 & 36 & Quiosques & SP \\
\hline Lopes et al. & 2016 & 3 & Comerciais & MG \\
\hline Skailo et al. & 2016 & 7 & Panificadora & PR \\
\hline Martins et al. & 2016 & 3 & Comerciais & PA \\
\hline Rudakoff $e t$ al. & 2018 & 30 & Institucionais & MA \\
\hline Ferro et al. & 2018 & 35 & Institucionais & TO \\
\hline Mendes et al. & 2019 & 2 & Institucionais & PI \\
\hline Carvalho et al. & 2018 & 10 & Comerciais & DF \\
\hline Santos et al. & 2018 & 10 & Quiosques & $\mathrm{BA}$ \\
\hline Ferreira et al. & 2017 & 8 & Institucionais & PA \\
\hline Lopes et al. & 2015 & 10 & Hotéis & $\mathrm{RS}$ \\
\hline Lyra et al. & 2017 & 8 & Comerciais & $\mathrm{ES}$ \\
\hline Silva et al. & 2017 & 292 & Ambulantes & AP \\
\hline Maia et al. & 2017 & 1 & Lanchonete & $\mathrm{CE}$ \\
\hline Costa et al. & 2017 & 14 & Ambulantes & $\mathrm{BA}$ \\
\hline Brandão et al. & 2020 & 16 & Institucionais & $\mathrm{AL}$ \\
\hline Teixera et al. & 2016 & 7 & UAN & ES \\
\hline Susin et al. & 2017 & 148 & UAN & $\mathrm{RS}$ \\
\hline Ramos et al. & 2020 & 20 & Institucionais & $\mathrm{RS}$ \\
\hline Ribeiro et al. & 2018 & 6 & Institucionais & SP \\
\hline Miranda et al. & 2012 & 5 & Comerciais & $\mathrm{BA}$ \\
\hline Carvalho et al. & 2017 & 5 & Comerciais & $\mathrm{RN}$ \\
\hline Fatel et al. & 2016 & 1 & UAN & RJ \\
\hline
\end{tabular}

*UF - Unidade da Federação: MA (Maranhão). RJ (Rio de Janeiro), RS (Rio Grande do Sul), MG (Minas Gerais), SP (São Paulo), PR (Paraná), PA (Pará), PI (Piauí), AL (Alagoas), AP (Amapá), DF (Distrito Federal), ES (Espírito Santo), CE (Ceará), BA (Bahia), TO (Tocantins), RN (Rio Grande do Norte). Fonte: Dados da pesquisa (2021). 
Quadro 2 - Adequação das condições higiênicas dos serviços de alimentação, de acordo com as categorias avaliadas. Brasil, 2021.

\begin{tabular}{|l|c|c|}
\hline \multicolumn{1}{|c|}{ CATEGORIAS } & Média (\%) & Variação min- Variação máx (\%) \\
\hline 1) Edificação, instalações, equipamentos, móveis e utensílios. & 59,84 & $4,12-95$ \\
\hline 2) Higienização de instalações, equipamentos, móveis e utensílios. & 66 & $20-100$ \\
\hline 3) Controle integrado de vetores e pragas urbanas & 38 & $22-100$ \\
\hline 4) Abastecimento de água & 86,7 & $20-90$ \\
\hline 5) Manejo de resíduos & 43 & $0-100$ \\
\hline 6) Manipuladores & 46 & $14-100$ \\
\hline 7) Matérias-primas, ingredientes e embalagens & 67,3 & $20-84$ \\
\hline 8) Preparação do alimento & 61,68 & $8,33-100$ \\
\hline 9) Armazenamento e transporte do alimento & 58,7 & $0-85$ \\
\hline 10) Documentação e registro & 38 & $0-100$ \\
\hline 11) Responsabilidade & 73 & $\mathbf{5 8 , 0 2}$ \\
\hline Geral & & \\
\hline
\end{tabular}

Fonte: Dados da pesquisa (2021).

Observou-se uma média de 58,02\% de adequação, onde se enquadrara no Grupo I apenas a categoria 4, se enquadraram no GRUPO II as categorias 1, 2, 7, 8, 9 e 11 e se enquadraram no GRUPO III as categorias 3, 5, 6 e 10. Apenas a categoria “Abastecimento de água” fez parte do grupo de maior adequação percentual (GRUPO I - 76\% A 100\%), obtendo o percentual de 86,7\% (Quadro 3).

Quadro 3 - Classificação dos grupos quanto ao percentual de adequações. Brasil, 2021.

\begin{tabular}{|c|l|}
\hline GRUPO 1 (76\%- 100\%) & \multicolumn{1}{|c|}{ Abastecimento de água } \\
\hline GRUPO 2 (51-75\%) & $\begin{array}{l}\text { Higienização de instalações, equipamentos, móveis e utensílios; } \\
\text { Matérias-primas, ingredientes e embalagens; Edificações, instalações, } \\
\text { equipamentos, móveis e utensílios Preparação do alimento; } \\
\text { Armazenamento e transporte do alimento preparado. }\end{array}$ \\
\hline Grupo 3 (< ou igual a 50\%) & $\begin{array}{l}\text { Controle integrado de vetores e pragas urbanas; Manejo de resíduos } \\
\text { Manipuladores; Documentação e registro. }\end{array}$ \\
\hline
\end{tabular}

Fonte: Dados da pesquisa (2021).

Encontrou-se uma variação muito grande nos percentuais ficando com menor desempenho na pesquisa as categorias que necessitam de registro documental tais como: documentos e registro; controle integrado de vetores e pragas e capacitação dos manipuladores, fator que demonstra a falta de empenho em cumprir com os requisitos legais estabelecidos, principalmente, nas creches e escolas públicas analisadas. Reflexo do ineficiente número de profissional nutricionista vinculado ao Programa Nacional de Alimentação Escolar - PNAE para atuação e fiscalização eficiente desses ambientes. O manejo de resíduos também obteve um baixo percentual de adequação devido à ausência de comprovação de controle de coleta do lixo e, principalmente, por não haver coleta seletiva do lixo em muitas das unidades. 


\section{Conclusão}

Em relação aos dados levantados por esta pesquisa pôde-se perceber um predomínio de produções científicas em instituições públicas. Observou-se também que a maioria das unidades de alimentação e nutrição avaliadas apresentam condições higiênico-sanitárias parcialmente adequadas (58,02\%) o que lhes classifica no Grupo 2 (51\% - 75\%). Os índices percentuais variaram bastante. As não-conformidades se fizeram predominantes nas seguintes categorias: manejo de resíduos, controle integrado de vetores e pragas, manipuladores e documentos e registros.

Constatou-se a necessidade de regularização documental em boa parte das unidades avaliadas. Nesse sentido, os resultados do estudo demonstram a necessidade de maior atenção dos órgãos de controle sanitário para com as unidades de alimentação e nutrição uma vez que a alimentação ofertada deve ser de qualidade nutricional e sanitária. Evidencia- se ainda a importância do nutricionista como responsável nos serviços de alimentação, pois a atuação desse profissional contribui com a adoção e manutenção das boas práticas no serviço de alimentação e, desta maneira, reduzindo os riscos de contaminações, surtos e doenças transmitidas por alimentos.

\section{Referências}

Almeida, E. B., Henn, R., Bartholo, S., \& Feder, D. (2016). Condições higiênico sanitárias de estabelecimentos de alimentação de um parque multitemático no Vale do Itajaí, Santa Catarina, Brasil. Segurança Alimentar e Nutricional, 23(2), 904-911.

Brasil. (2002). Ministério da Saúde. Resolução - RDC N²75/ 2002. Dispõe sobre o Regulamento Técnico de Procedimentos Operacionais Padronizados aplicados aos Estabelecimentos Produtores/ Industrializadores de Alimentos e a Lista de Verificação das Boas Práticas de Fabricação em Estabelecimentos Produtores/ Industrializadores de Alimentos. Diário Ofical da União, Brasília.

Brasil. (2004). Ministério da Saúde. Agência Nacional de Vigilância Sanitária. Resolução RDC nº 216 , de 15 de setembro de 2004 . Regulamento Técnico de Boas Práticas de Serviços de Alimentação. Brasília.

Brasil. (2014). Ministério da Saúde. Agência Nacional de Vigilância Sanitária. Resolução no 52 , de 24 de setembro de 2014 . Dispõe Sobre o Regulamento Técnico de Boas Práticas para os Serviços de Alimentação. Diário Oficial da República Federativa do Brasil, Poder Executivo, Brasília, DF. Seção 1, p.51.

Brandão, T. B. C., Padilha, B. M., Campos, E. L. L., Severiano, G. M. L., \& Torres, J. C. L. Adequação numérica de nutricionistas e condições higiênicosanitárias de unidades de alimentação e nutrição escolares de Maceió, Alagoas. Segurança Alimentar e Nutricional, 27, 1- 8, 2020.

Aguiar, O. B., Kraemer, F. B., \& Menezes, M. F. G. (2019). Gestão de Pessoas em Unidade de Alimentação e Nutrição. Rubio. Associação Brasileira das Empresas de Refeições Coletivas (Aberc). Mercado Real de Refeições. http://www.aberc.com.br/mercadoreal.asp.

Almeida, G. L., Costa, S. R. R., \& Gaspar, A. (2012). A Gestão da Segurança dos Alimentos em Empresa de Serviço de Alimentação e os Pontos Críticos de Controle dos Seus Processos. Boletim CEPPA, 30(1), 135-146p.

Azeredo, D. R. P. (2017). Inocuidade dos Alimentos. Atheneu.

Carvalho, C. T., Sousa, C. P., Fernandes, S. R. S., Silva, T. C., Silva, T. M., \& Luz, J. R. D. (2017). Condições higiênico sanitárias da carne bovina vendida em feiras livres de Natal, Rio Grande do Norte. Nutrivisa, (3)3, 121- 129.

Carvalho, E. S., Sousa, D. S., Ferreira, A. M., \& Dourado, L. P. S. (2018). Condições higiênico-sanitárias de food trucks localizados em instituições de ensino superior privadas do Distrito Federal. Hig. Alimentar, 32, 284-285.

Cintra, P. (2016). Qualidade e Redução de Custos em Alimentos. Rubio.

Costa, C. P., \& Silva, E. A. Jr. (2016). Condições higiênico das barracas de alimentos prontos, comercializados nas feiras de arte, artesanato e antiguidades da subprefeitura da Sé em São Paulo. Hig. Alimentar, 30, 50 - 54.

Costa, M. C., Damascena, S. S., Silva, J. N., \& Silva, I. M. M. (2017). Condições higiênico de food trucks em uma cidade do Recôncavo da Bahia. Magistra, 29, 328-334.

Fatel, E. C. S., et al. (2016). Avaliação das Condições Higiênico-sanitárias de Restaurante Universitário antes e após a Implantação das Boas Práticas de Produção. Higiene Alimentar, 21(136), 35-39.

Ferreira, A. C. C., Morais, B. H. S., \& Rezende, A. L. S. (2017). Estudo comparativo das condições higiênico sanitárias em unidades de nutrição e dietética em Belém, Pará. Nutrivisa, 3(3), 129-136.

Ferro, L. L., Fialho, C. J., Pires, C. R. F., Teles, N. B., \& Santos, V. F. (2018). Condições higiênico-sanitárias de unidades de alimentação e nutrição de escolas públicas do estado do Tocantins. Segurança Alimentar e Nutricional, 25, 118-130.

Figueiredo, R. P., \& Ribeiro, M. C. S. (2013). Avaliação das condições higiênico-sanitárias de cantinas de escolas particulares em São Luis, MA. Hig. Alimentar, 27, 216-227. 
Fiori-Stangarlin, L., Serafim, A. L., \& Sacool, A. L. F. (2016). Instrumentos para Elaboração do Manual de Boas Práticas e dos Procedimentos Operacionais Padronizados em Serviços de Alimentação. Rubio.

Fonseca, K. Z., \& Santana, G. R. (2012). Guia Prático para Gerenciamento de Unidade de Alimentação e Nutrição, UFRB, 62.

Germano, P. M. L., \& Germano, M. I. S. (2011). Higiene e vigilância sanitária de alimentos, Manole, 1088.

Gobis, M. A., \& Campanatti, R. (2012). Os benefícios da aplicação de ferramentas de gestão de qualidade dentro das indústrias do setor alimentício, Revista Hórus, 1, 26-39.

Gomes, J. A., Silveira, J. T., Moura, F. A., \& Almeida, L. C. (2016). Avaliação das condições higiênicas de uma unidade de alimentação e nutrição de uma organização militar, Higiene Alimentar, 30, 61 - 65.

Gomes, R. N., Santos, C. N. C., \& Lago, E. C. (2016). Adequação condições higiênico-sanitária de uma unidade de alimentação de escola pública em município do Maranhão, Higiene Alimentar, 30, 260 - 265.

Jucene, C. (2019). Manual de Segurança Alimentar: boas práticas para os serviços de alimentação. (3a ed,), Rubio.

Jucene, C. (2014). Plano APPCC em estabelecimentos alimentícios: guia técnico para elaboração. Rubio.

Lopes, L. L., Silveira, J. T., \&| Floriano, J. M. (2015). Condições higiênico-sanitárias de hotéis em Uruguaiana, Rio Grande do Sul- RS. Nutrivisa, 2 , 16 - 21.

Lopes, N. M., \& Ferreira, L. C. (2016). Avaliação da higienização e sanitização em açougues da cidade Januária, MG. Higiene Alimentar, 30,262 - 263.

Lima, M. B., Saturnino, C. M. M., \& Tobal, T. M. (2017). Avaliação da adequação das boas práticas de fabricação em serviços de alimentação. Rev. Inst. Adolfo Lutz, 76(1), 1722- 1729.

Lyra, G. R., Motta, M. C. M., \& São José, J. F. B. (2017). Avaliação das condições higiênico-sanitárias em restaurantes comerciais em Vitória- ES. Rev. Inst. Adolfo Lutz, 76(1), 1722- 1729.

Maia, M. O. (2017). Avaliação das condições higiênico-sanitárias de uma lanchonete no município de Limoeiro do Norte- CE. Revinter, 10(1), 45-46.

Martins, D. A., et al. (2016). Avaliação das condições higiênico-sanitárias em pizzarias na cidade de Marabá- PA. Scientia Plena, 12 , 37 - 41.

Mendes, K. D. S., Silveira, R. C. P., \& Galvão, C. M. (2008). Revisão integrativa: método de pesquisa para a incorporação de evidências na saúde e na enfermagem. Texto e contexto - Enfermagem, 17, 758-764.

Mendes, T. I. L., Monteiro, M. L. S., Carvalho, L. M. F., \& Bezerra, K. C. B. (2019). Condições higiênico-sanitárias em unidades de alimentação em escolas de tempo integral. Revista Eletrônica Acervo Saúde, 31, 37- 41.

Miranda, P. C., \& Barreto, N. S. E. (2012). Avaliação higiênico sanitária de diferentes estabelecimentos de comercialização da carne-de-sol no município de Cruz das Almas - BA. Revista Caatinga, Natal, 25(2), 166-172.

Pereira, A. S., Shitsuka, D. M., Parreira, F. J., \& Shitsuka, R. Metodologia da pesquisa científica. UFSM. https:// repositorio.ufsm.br/bitstream/handle/ 1/15824/Lic_Computacao_MetodologiaPesquisa-Cientifica.pdf?sequence=1.

Ramos, C. I., Valença, M. S., Peter, N. B., \& Muniz, L. C. (2020). Avaliação das boas práticas de unidade de alimentação escolar da zona rural do município de Pelotas - RS. Semina: Ciências Biológicas e da Saúde, 41, 67- 74.

Ribeiro, J. A., Damasceno, K. J. L., Moura, K. D. L., Salvador, A. A., Rosseti, F. X., Tamasia, G. A., Bello, S. R. B., \& Vicentinni, M. S. (2018). Análise das condições higiênico sanitárias das unidades de alimentação e nutrição das escolas de m município no Vale do Ribeira, SP. Research, Society and Development, 7,327 a 348 .

Rudakoff, L. C. S., Mouchreck, A. N., Frota, M. T. B. A., \& Bastos, L. S. (2018). Qualidade e segurança alimentar em unidades de alimentação e nutrição escolares da rede municipal de educação de São Luis, Maranhão. Vigilância Sanitária em Debate, 6, 46- 53.

Sacool A. L. F., Stangarlin L., \& Hecktheuer L. H. (2012). Instrumentos de Apoio para Implantação das Boas Práticas em Empresas Alimentícias. 1 Ed, Rio de Janeiro: Rubio.

Santos, J. M., \& Ferreira, L. C. (2019). Condições higiênico-sanitárias de uma escola pública no município de Januária- MG antes e após o treinamento dos manipuladores de alimentos. Cad. Ciências Agrárias, 11, 01-07.

Santos, et al. (2018). Avaliação da aplicação de boas práticas na comercialização de água de coco em quiosques localizados em Salvador- BA. Hig. Alimentar, $32,46-51$.

Silva, A. C. Jr.; Ferreira, L.; \& Frazão, A. (2017). Avaliação da condição higiênico-sanitária da comercialização de pescados na feira do produtor rural de Buritizal, Macapá- AP. Life Style, 4, 71-81.

Silva L. C. et al. (2015). Boas Práticas na Manipulação de Alimentos em Unidades de Alimentação e Nutrição. Demetra: Alimentação, nutrição e saúde, $10(7), 797-820$

Soares, D. S. B., et al. (2018). Boas Práticas na Manipulação de Alimentos em Unidades de Alimentação e Nutrição Escolares em um Município do Estado do Rio de Janeiro- Brasil. Ciência e Saúde Coletiva, 23(12), 4077-4083.

Skailo, M.; \& Machado, A. D. (2017). Condições higiênico-sanitárias em produção de alimentos em panificadoras de dois municípios do sudoeste paranaense. Hig. Alimentar, 31, 274-275. 
Research, Society and Development, v. 10, n. 14, e185101422070, 2021

(CC BY 4.0) | ISSN 2525-3409 | DOI: http://dx.doi.org/10.33448/rsd-v10i14.22070

Susin, V., et al. (2017). Condições higiênico-sanitárias, estruturais e de funcionamento em unidade de alimentação e nutrição. Vig. Sanit. Debate, 5, 60-68.

Rodrigues, K. L. (2010). Segurança Alimentar em Unidades de Alimentação e Nutrição. 148 f. Tese (Doutorado em Ciências e Tecnologia Agroindustrial) Universidade Federal de Pelotas, Pelotas.

Teixeira, A. C., et al. (2016). Boas práticas na manipulação de alimentos em restaurantes comerciais em self service. Hig. Alimentar, $30,258-259$.

Vasconcelos, R. M. (2017). Condições higiênico-sanitárias na produção de alimentos em creches públicas na cidade do Rio de Janeiro - RJ. Hig. Alimentar, 31, 264-265.

Vidal, G. M., et al. (2011). Avaliação das boas práticas em segurança alimentar de uma unidade de alimentação e nutrição de uma organização militar da cidade de Belém, PA. Alim. Nutr. Araraquara, 22, 283-290. 